\title{
Sedation and analgesia in critically ill patients with COVID-19: a cohort retrospective study
}

\author{
Caterina Aurilio ${ }^{1, \star}$, Antonio Corcione ${ }^{2}$, Fiorentino Fraganza ${ }^{3}$, Pasquale Sansone ${ }^{1}$, \\ Antonella Paladini ${ }^{4}$, Maria Beatrice Passavanti ${ }^{1}$, Vincenzo Pota ${ }^{1}$, \\ Francesco Coppolino ${ }^{1}$, Francesca Molino ${ }^{1}$, Maria Caterina Pace ${ }^{1}$
}

\author{
${ }^{1}$ Department of Woman, Child and \\ General and Specialized Surgery, \\ University of Campania "Luigi Vanvitelli", \\ 80011 Naples, Italy \\ ${ }^{2}$ Intensive Care Unit, AORN Ospedali dei \\ Colli-Monaldi Hospital, 80133 Naples, \\ Italy \\ ${ }^{3}$ Cotugno Hospital, AORN Ospedali dei \\ Colli, 80133 Napoli, Italy \\ ${ }^{4}$ Department MESVA, University of \\ L'Aquila, 67100 L'Aquila, Italy \\ *Correspondence \\ caterina.aurilio@unicampania.it \\ (Caterina Aurilio)
}

\begin{abstract}
Introduction: The global pandemic of novel coronavirus disease 2019 (COVID-19) began in Wuhan, China in December 2019 and spread worldwide. This retrospective study summarizes clinical experience and analgo-sedation treatment used for this novel epidemic in an Italian ICU between February to May 2020.

Methods: This is a retrospective trial of 72 patients with laboratory-confirmed SARSCoV-2 infection (COVID-19) admitted to ICU between February15, 2020 and May 15, 2020. Main Outcomes are demographic, clinical data (age, sex, medical comorbidities, respiratory supports, positive end-expiratory pressure values (PEEP), fraction of inspired oxygen $\left(\mathrm{FiO}_{2}\right)$, arterial partial pressure of oxygen $\left(\mathrm{PaO}_{2}\right)$, sedatives and analgesic drugs, their dosage and way of administration, medical treatments and patients mortality), ventilation strategies, sedation management in ICUs and patient mortality.

Results: The mean age of the 72 patients included in the study was 69 years (SD \pm 12 ), $77 \%$ were male. All patients required respiratory support and sedation management according to different techniques of ventilation: $100 \%$ received deep sedation (RASS 4) before intubation and invasive mechanical ventilation and 19\% received light sedation (RASS -1) and noninvasive ventilation).

Conclusions: Of the 72 patients admitted in ICUs, 92\% died. A total of 608 patients admitted to the AORN dei Colli "Naples" with confirmed COVID-19. In this restrospective study we have analyzed 72 (out of 608) patients that were admitted to intensive care due to worsening clinical conditions. All patients required tracheal intubation and mechanical ventilation with deep sedation (RASS -4), while only $19 \%$ (patients required light sedation so RASS -1) were subsequentely swiched to light sedation because of the need of compliance with non-invasive ventilation. Sedation was obtained with propofol, remifentanil, midazolam and dexmedetomidine, according to hospital guidelines.
\end{abstract}

\section{Keywords}

COVID-19; Naso-pharyngeal swab; Pneumonia; Acute respiratory failure; Interstitial lung disease; Sedation

\section{Introduction}

The novel coronavirus disease 2019 (COVID-19) began in Wuhan, China in December 2019, since then, it has spread fast worldwide [1]. On March 2020 the World Health Organization (WHO) declared the severe acute respiratory syndrome coronavirus (SARS-CoV-2) outbreak a pandemic disease [2].

COVID-19 is complex disease that requires hospitalization in ICUs when clinical condition worsen [3]. Many organs are involved in the infection. The main target is the lung with phenomena of pneumonia, atelectasis, hypo/hyperperfused areas, thromboembolic events and consolidation, that, when they occur, require hospitalization in intensive care [4]. Patients with SARS-CoV-2 can also present, in addition to an acute respiratory syndrome, other critically manifestations [5]. In the central nervous system we can witness impaired consciousness, thrombotic events, ischemic/hemorragic stroke meningoencephalitis, Guillain-Barre' syndrome [6]. Also kidney may present injury which frequently needed dialisys [7].

Besides oxygenation and optimal hydro-electrolytic homeostasis, sedation and pain management are objectives of the management of critically ill patients admitted to ICUs and under mechanical ventilation both invasive or non-invasive [8].

Pain control should be a first priority in the care of critically ill patients to facilitate specific interventions such as 
management of intracranial pressure, prone position, neuromuscular blockade and controlled mechanical ventilation [9]. Pain therapy is mainly based on intravenous analgesics, like opioids and non-opioid agents in a classical multimodal analgesia, in order to minimize opioids side effects $[10,11]$. The 2018 Pain, Agitation/sedation, Delirium, Immobility and Sleep (PADIS) guidelines attempt to this mission by updating the 2013 Pain, Agitation, and Delirium (PAD) guidelines. The 2013 PAD guidelines suggest that non benzodiazepine sedatives are preferable to benzodiazepine sedative in critically ill mechanically ventilated adults because of short-term outcomes, such as ICU long stay, duration of mechanical ventilation and delirium.

The recommendation of PADIS suggests using either propofol or dexmedetomidine over benzodiazepines for sedation in critically ill mechanically ventilated adults. If pain management is not completely adequate to patient's need, light or deep sedation can be necessary. The light sedation is often measured with the Richmond Agitation-Sedation Scale (RASS Scale) with values vary from -2 to 0 . The deep sedation RASS Scale (from -5 to -3 ), is required to tolerate mechanical ventilation and limit the risk of ventilator lung injury. In the COVID-19 pandemic, patients need more aggressive ventilatory management that require deep sedation, propofol and dexmedetomidine may be used as the primary sedative agents [12]. Severe COVID-19 patients may require continuous neuromuscular blocking agents (NBMA) to optimize mechanical ventilation [13].

The rationale for this cohort retrospective study is particularly relevant during the ongoing COVID-19 pandemic due to the recent concerns about sedation treatment. We analyzed analgo-sedation treatment in the initial case series of patients hospitalized in our ICUs.

We report the results of a validated and reliable sedation assessment tools, the Richmond Agitation-Sedation Scale (RASS) used for sedation assessment and evaluate the sedation management of critically ill patients admitted in ICUs [14].

\section{Methods}

This is a retrospective observational case study, analyzing the patients recovered in the ICU from February 15, 2020 to May 15, 2020. All the patients with laboratory-confirmed SARSCoV-2, by RT-PCR, hospitalized in the department of infectious disease, who were admitted to ICUs for the worsening of their conditions when $\mathrm{SpO}_{2}$ was $<90 \%$ or $\mathrm{PaO}_{2} / \mathrm{FiO}_{2}<150$ or $\mathrm{RR}>28 / \mathrm{min}$, low Glasgow coma score $<8$, hemodynamic instability, lactate level $>3 \mathrm{mEq} / \mathrm{L}$. All the other patients SARS-CoV-2 positive admitted to the other department and sub-intensive care unit (SICU) were excluded from the data collection.

This retrospective analysis is based on data from medical records for the evaluation of patients affected by SARS-CoV2 infection and treated with standard clinical therapy, and so ethics committee approval was not required. All patients or their legal representatives provided written informed consent for data collection. It was conducted in accordance with the International Conference on Harmonisation Good Clinical Practice guidelines and the provisions of the 2008 Declaration of Helsinki. Patient approval or their legal representatives for the publication was obtained.

Data were extracted by the medical records on a case report form (CRF) and transferred to electronic excel sheet.

The collected data included: age, sex, medical comorbidities, respiratory supports, positive end-expiratory pressure values (PEEP), fraction of inspired oxygen $\left(\mathrm{FiO}_{2}\right)$, arterial partial pressure of oxygen $\left(\mathrm{PaO}_{2}\right)$, sedatives and analgesic drugs, their dosage and way of administration, medical treatments and patients mortality. In the 72 patients admitted to ICUs the sedation level was measured using the RASS scale.

No statistical sample size calculation was performed a priori and sample size was equal to the number of patients treated during the study period. Categorical variables are expressed as number of patients (percentage) with $95 \%$ CIs. Percentages of available data for overall population are based on the total number of patients included in the study, distribution of available data over the age subgroups are based on the available data for that variable, and the other percentages are calculated using the number of available data for that subgroup.

\section{Results}

Between February 21st and May 15th, 2020, 608 patients were admitted to the Hospital with confirmed COVID-19. Of them, $72(11.8 \%)$ were transferred to the Intensive Care Unit (ICU). All patients legal representatives provided written informed consent for data collection. Out of those patients, $77 \%$ were male and the mean age was 69 (SD +/- 12) years. Overall $65 \%$ were over 65 years old and the remaining $32 \%$ were over 75 years old. Patients with no comorbidities were $20 \%, 15 \%$ had only one comorbidity and $65 \%$ had two or more comorbidities. The most common comorbidities were hypertension (27\%) cardiovascular disease (18\%) followed by chronic kidney disease $(16 \%)$, diabetes and dyslipidemia (15\%), chronic lung disease (14\%) and obesity (10\%) (Fig. 1)

All patients admitted to the ICU required invasive mechanical ventilation, $19 \%$ of them showed a respiratory improvement and were weaned from mechanical ventilation, extubated and during the extubating period, treated with non-invasive positive pressure ventilation (NIPPV) during their ICU stay as respiratory support. The number of patients $11.8 \%$ (72) who needed invasive mechanical ventilation was similar compared with data reported by other studies [15]. All patients were treated with protective ventilation. The median PEEP was $12 \mathrm{~cm}$ of $\mathrm{H}_{2} \mathrm{O}$ and the median $\mathrm{FiO}_{2} 0.8$ with no significant substantial differences between ages. All these patients were treated in ICU and the COVID-19 patients infected, requiring non-invasive ventilation were managed outside the ICU in the pulmonary unit; furthermore, data of these patients were not recorded in ICU.

According to previous WHO guidelines, at the time of analysis, drug treatments for COVID-19 in the cohort of ICU hospitalized patients consisted in anti-inflammatory (corticosteroids), anticoagulant, antiviral agents, monoclonal antibodies (only in the first days of onset of infection) protective respiratory support [16]. Corticosteroids may lead to decrease the host inflammatory responses in the lungs, however glucocorticoids have not been routinely administered in the 


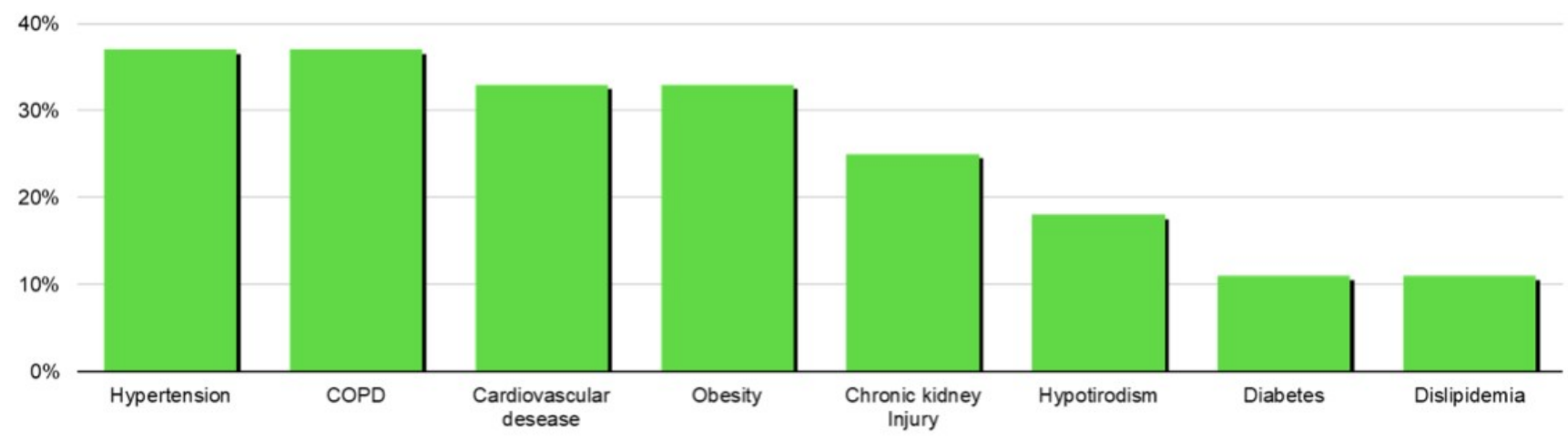

F I G U R E 1. The most common comorbidities of the patients.

patients, methylprednisolone $40 \mathrm{mg}$ daily was administered to the $51 \%$ of the patients, except an early use $(17 \%)$ for patients with severe symptoms of ARDS at the admission in ICU, in agreement with $\mathrm{WHO}$, unless there was a separate evidencebased indication [17].

In ICU patients who required deep sedation were treated with continuous infusion of propofol $70-250 \mathrm{mg} / \mathrm{h}$, continuous infusion of dexmedetomidine with a mean dosage of 0.8 to $1.5 \mu \mathrm{g} / \mathrm{kg} / \mathrm{h}$ as adjuvant sedative for deep sedation, for the management of weaning and non-invasive ventilation in patients in the first hours post-extubating $[18,19]$. Remifentanil $0.05-0.02 \mu \mathrm{g} / \mathrm{kg} / \mathrm{min}$, was administered for pain management in intubated patients in mechanical ventilation [20]. Only $19 \%$ of the patient population was treated with NIPPV. It was used as management of respiratory weaning during the first hours post-extubating. All those patients needed a light sedation. It was performed with continuous infusion of propofol alone in $40 \%$ of patients with a mean dosage of $10-50 \mathrm{mg} / \mathrm{h}$, continuous infusion of dexmedetomidine in $20 \%$ of patients with a mean dosage of 0.2 to $0.7 \mu \mathrm{g} / \mathrm{kg} / \mathrm{h}$ and $40 \%$ of patients with the association of propofol $10-50 \mathrm{mg} / \mathrm{h}$ and dexmedetomidine 0.2 to $0.7 \mu \mathrm{g} / \mathrm{kg} / \mathrm{h}$, with sedation evaluated using RASS scale sedation $(-1.8 \pm 0.94)[21]$.

Neuromuscular blockade drugs were used in all patients in order to manage the mechanical ventilation strategy (recruitment, high PEEP) as well prone positioning was used in $22 \%$ according to the recent literature on ARDS management [22, 23]. Rocuronium bromide was the neuromuscular blockade used (30\% of patients in mechanical ventilation) and was administered in continuous infusion, at the rate of $8 \mathrm{mg} / \mathrm{h} \mathrm{[24].}$

All critically ill patients admitted to the ICU were evaluated for severity and differential diagnosis of lung disease using computed tomography (CT). Lung and cardiac ultrasonography were performed at the bed side of the entire patient population, as well as a basic electrocardiogram (ECG) and 12lead ECG, which is important for patients on QT-prolonging drugs.

Out of the 72 patients admitted in ICUs 92\% died. In ICU were admitted only patient with lung failure and need of invasive mechanical ventilation with very low $\mathrm{PaO}_{2} / \mathrm{FiO}_{2}$ ratio $(<100)$ or presence of Shock with the need of vasoactive drugs. Respiratory failure was a main cause of death. $83 \%$ percent of patients died from respiratory failure, $9 \%$ from cardiovascular failure, 3\% from cerebral thrombosis/haemorrhage 5\% from hepato-renal failure. These data are in agreement with national data for the same period of time [5].

\section{Discussion}

We report a cohort of critically ill patients, that had serious and often fatal pneumonia, admitted to ICUs of the designated hospitals in Naples, with laboratory-confirmed COVID19 from February 15 to May 15, 2020. Out of the 72 patients admitted in ICUs $92 \%$ died. Overall $65 \%$ were over 65 years old and the remaining $32 \%$ were over 75 years old. In ICU were admitted only patient with lung failure and need of invasive mechanical ventilation with very low $\mathrm{PaO}_{2} / \mathrm{FiO}_{2}$ ratio $(<100)$ or presence of Shock with the need of vasoactive drugs. A recent systematic review and meta-analysis of outcomes of patients admitted to ICU with COVID-19 demonstrated, more over, that as the pandemic has progressed, the reported mortality rates have reduced from above 50\% in March 2020 to close to $40 \%$ at the end of May 2020 worldwide [25]. The analysis of mortality during an outbreak is not easy and precise to do and so a correct evaluation can be obtained only once the outbreak is over. Furthermore, the high Italian mortality may well be attributable to a large proportion of elderly persons in the Italian population, to an ascertainment bias and/or diagnosis bias, leading to an underestimation of the milder cases and mortality overestimation [26].

The agent causing the infection was assessed to be a coronavirus, depending on RNA viral synthesis, causing a high number of cases of severe acute respiratory syndrome due to a disrupted immune response or "cytokine storm" [27].

Several medications used for other diseases have been tested for treating the SARS-CoV-2 infection from moderate to severe cases, though there has yet to be clear evidence. The combination of Lopinavir and Ritonavir, a protease inhibitor for the treatment of HIV-1 infection and effective against MERS-CoV, on the success of these therapy and on the recommendation of the WHO, was tried in the treatment of SARS-CoV-2 [28]. In the cohort of patients admitted to ICUs Cotugno Hospital $36 \%$ received Lopinavir/Ritonavir. Remdesivir is another antiviral drug, which inhibits RNA synthesis, founded for the treatment of Ebola infection [29]. On 1th May 2020 the U.S. 
Food and Drug Administration authorized the use of Remdesivir for COVID-19 patients. $24 \%$ of patients recovered in ICUs Hospital Cotugno received Remdesivir. Besides antiviral drugs and other medications, as potential anti-infective agents have been proposed for the treatment of COVID-19. Among the anti-infective agents repurposed to treat COVID-19 the Hydroxychloroquine, according to different reports, possess antiviral activity against several virus. Hydroxychloroquine inhibits viral entry and endocytosis and has been used for the treatment and prophylaxis of malaria and rheumatic desease for more than 50 years. In March 2020 the WHO approved the compassionate use of hydroxychloroquine in hospitalized patients with COVID-19 [30]. 92\% of patients recovered in ICUs Hospital were treated with hydroxychloroquine and $15 \%$ received at the same time Azitromycin. Azitromycin could inhibit endocystosis of the virus and its replication. Monoclonal antibodies directed against inflammatory cytokines represent another adjunctive therapies for COVID-19. Their use is licensed for the damage in the lungs and other organs caused by an amplified immune response and cytokine releases, like IL-6 [31]. Tocilizumab is a monoclonal antibody against the interleukin-6-receptor (IL-6R) and the Italian Medicine Agency (AIFA) approved the use of Tocilizumab in patients with COVID-19. The Cotugno Hospital in Naples has been the first Hospital in Italy to experiment this medicine in a III phase of experimentation in small series of severe COVID19 cases with early report of success. Disseminated intravascular coagulopathy and D-didimer were found to be higher than in control healthy population than in COVID-19 population. Hypercoagulation associated with COVID-19 may be due to increased angiotensin II expression and consequently increased plasminogen activator inhibitor $\mathrm{C}-1$ expression, which is consistent for reduced fibrinolysis [32]. Anticoagulant therapy mainly with low molecular weight heparin appears to be associated with better prognosis in severe COVID-19 and has been recommended by some expert consensus because of the risk of disseminated intravenous coagulation [33]. 14\% were treated with enoxaparine in Cotugno Hospital. Among adjunctive therapies in the ICUs patients the corticosteroids may lead to decrease the host inflammatory responses in the lungs. Glucocorticoids have not been routinely administered in the patients admitted in the ICUs Cotugno Hospital, except an early use for patients with severe symptoms of ARDS at the admission in ICU, in agreement with WHO, unless there was a separate evidence-based indication [34].

Among critically ill patients distress due to pain is common. The use of drugs for sedation in critically ill patients requires a flexible approach to reduce the incidence of adverse effects [35]. There is a relationship between ventilatory and sedation management and the priorities should be to handle increased respiratory drive, to optimize ventilation and to treat ventilator dysynchrony [36]. Furthermore, the primary reasons for sedation in COVID-19 patients receiving lung protective ventilation include: improving patient comfort such as pain, anxiety and dyspnea [37], enhancing patient safety during special maneuvers such as pronate and maintaining patient interaction with staff and family or promote early physical and cognitive recovery [38]. All patients were treated with protective ventilation. The median PEEP was $12 \mathrm{~cm}$ of $\mathrm{H}_{2} \mathrm{O}$ and the median $\mathrm{FiO}_{2} 0.8$ with no significant substantial differences between ages. The ventilation strategies was based on the evidence on the treatment of ARDS. The $\mathrm{PaO}_{2} / \mathrm{FiO}_{2}$ thresholds was used for categorizing patients with respect to different therapeutic approaches. The median Peep was 12 due to very low $\mathrm{PaO}_{2} / \mathrm{FiO}_{2}$ ad the admission. The decision to add neuromuscular blockade or to proceed to pronation was based on the worsening of $\mathrm{PaO}_{2} / \mathrm{FiO}_{2}$ as on our common clinical practice in the management of severe ARDS [24].

Distress needs to be treated to diminish sympathetic tone and agitation which may occur in critically ill patients and is often the cause of ventilator asynchrony and vital sign abnormalities. The best clinical practice recommendations [39, 40] indicate analgesia in case of impaired pulmonary compliance, severe ventilator asynchrony and distress [41].

To optimize the use of sedatives, opioids, and neuromuscular blocking agents the patients should be considered for an individual management of sedation and analgesia, adjustable over time, reaching the lightest level of sedation and giving priority to pain control [42]. The use of opioids, morphine sulfate, fentanyl, sufentanil and remifentanil (with the advantage of a rapid onset of action and prompt clearance, independent of hepatic and renal function,) may achieve optimal analgesia in most patients [43]. Also, non-opioids, that include paracetamol, ketamine $(1-2 \mathrm{mcg} / \mathrm{kg} / \mathrm{min})$, lidocaine $(1 \mathrm{mg} / \mathrm{kg} / \mathrm{h})$ magnesium sulphate $(0.08-0.16 \mathrm{gr} / \mathrm{h})$ with different mechanisms of action may achieve a beneficial effect on opioids side effects. If cough reflex occurs during intubation, volatile sedation, with inhalational anesthetics desflurane or sevoflurane [44], should be a strategy for its treatment. Volatile sedation should be used, furthermore, to spare adverse effects of opioids such as prolonged sedation, hallucinations, hyperalgesia and immunosuppression [44]. The deep sedation is required to tolerate mechanical ventilation and limit the risk of ventilatorinduced lung injury. Therapeutic modalities should include propofol or benzodiazepines and dexmedetomidine in case of propofol-related infusion syndrome and intermittent use of short-term muscle relaxation (cis-atracurium, atracurium, rocuronium 24-48 h) rather than continuous, for persistent diaphragmatic weakness, [45]. Opioid analgesics are the common sedative-analgesic medications used in critically ill patients and are most commonly used to manage distress due to pain in mechanically ventilated patients. The multimodal analgesia, with different drugs with different mechanisms of action, may help to avoid delays in ventilator dismission, to decrease the use of analgesics, the deep sedation and better facilitate ventilator weaning [46, 47].

The light sedation can be achieved with propofol, continuous or intermittent infusion of dexmedetomidine ( 0.2 to 1.4 $\mathrm{mcg} / \mathrm{kg} / \mathrm{h}$ with no bolus) and is associated with strategy of mechanical ventilation weaning [48].

The Behavioral pain scale (BPS) and the Critical Care Pain Observational Tool (CPOT), if the patient is unable to selfreport, are the most commonly used scale [49]. The Richmond Agitation Sedation Scale (RASS) and the NRS are the scale used, if patient is able to self-report. If BPS $>6$, CPOT $>2$ and NRS $>4$ the patient is in significant pain $[50,51]$.

The first priority for intensivists is optimize patient comfort avoiding over-sedation which may induce a longer duration of 
mechanical ventilation and ICU stay [52].

\section{Limitations}

This study has several limitations. First: the study was a retrospective evaluation at the early stage of COVID-19 pandemic in Naples. Second: the study population was limited to those who were only admitted to ICUs. Third: no control group and the conclusions may not be generally applicable to other populations.

\section{Conclusions}

Pain control and patient comfort are the first objectives for sedation in COVID-19 patients to improve anxiety, to reduce the metabolic and cardiovascular effects of pain-induced sympathetic activity.

In this study, out of 72 critically ill patients with laboratoryconfirmed COVID-19 admitted to ICUs overall of patients have been managed with sedation and analgesia to achieve better ventilation and prevent ventilatory dysynchrony.

\section{AUTHOR CONTRIBUTIONS}

$\mathrm{AC}, \mathrm{FF}, \mathrm{FC}, \mathrm{FM}$, conducted the research, CA, MCP, designed research study, CA, MCP, PS, VP wrote the manuscript, MBP, AP analyzed data. All authors contributed to editorial changes in the manuscript. All authors read and approved the final manuscript.

\section{ETHICS APPROVAL AND CONSENT TO PARTICIPATE}

This retrospective analysis is based on data from medical records for the evaluation of patients affected by SARS-CoV2 infection and treated with standard clinical therapy, and so ethics committee approval was not required. All patients or their legal representatives provided written informed consent for data collection. It was conducted in accordance with the International Conference on Harmonisation Good Clinical Practice guidelines and the provisions of the 2008 Declaration of Helsinki.

\section{ACKNOWLEDGMENT}

Thanks to all the peer reviewers for their opinions and suggestions.

\section{FUNDING}

This research received no external funding.

\section{CONFLICT OF INTEREST}

The authors declare no conflict of interest.

\section{REFERENCES}

[1] Lai C, Shih T, Ko W, Tang H, Hsueh P. Severe acute respiratory syndrome coronavirus 2 (SARS-CoV-2) and coronavirus disease-2019
(COVID-19): the epidemic and the challenges. International Journal of Antimicrobial Agents. 2020; 55: 105924.

[2] Park SE. Epidemiology, virology, and clinical features of severe acute respiratory syndrome -coronavirus-2 (SARS-CoV-2; Coronavirus Disease-19). Clinic Experimetal Pediatrics. 2020; 63: 119-124.

[3] Li X, Ma X. Acute respiratory failure in COVID-19: is it "typical" ARDS? Critical Care. 2020; 24: 198.

[4] Gattinoni L, Chiumello D, Rossi S. COVID-19 pneumonia: ARDS or not? Critical Care. 2020; 24: 154.

[5] Grasselli G, Zangrillo A, Zanella A, Antonelli M, Cabrini L, Castelli A, et al. Baseline Characteristics and Outcomes of 1591 Patients Infected with SARS-CoV-2 Admitted to ICUs of the Lombardy Region, Italy. Journal of the American Medical Association. 2020; 323: 1574-1581.

[6] Sansone P, Giaccari LG, Aurilio C, Coppolino F, Esposito V, Fiore M, et al. Post-Infectious Guillain-Barré Syndrome Related to SARS-CoV-2 Infection: A Systematic Review. Life. 2021; 11: 167.

[7] Guan WJ, Ni Y, Hu Y, Liang WH, Ou CQ, He JX, et al. Clinical characteristics of coronavirus disease 2019 in China. New England Journal of Medicine. 2020; 387: 1708-1720.

[8] Foti G, Giannini A, Bottino N, Castelli GP, Cecconi M, Grasselli G, et al. Management of critically ill patients with COVID-19: suggestions and instructions from the coordination of intensive care units of Lombardy. Minerva Anestesiologica. 2020; 86: 1234-1245.

[9] Chanques G, Constantin J, Devlin JW, Ely EW, Fraser GL, Gélinas C, et al. Analgesia and sedation in patients with ARDS. Intensive Care Medicine. 2020; 46: 2342-2356.

[10] Payen JF, Chanques G, Futier E, Velly L, Jaber S, Constantin JM. Sedation for critically ill patients with COVID-19: which specificities? One size does not fit all. Anaesthesia, Critical Care \& Pain Medicine. 2020; 39: 341-343.

[11] Chanques G, Drouot X, Payen J. 2008-2018: Ten years of gradual changes in the sedation guidelines for critically ill patients. Anaesthesia, Critical Care \& Pain Medicine. 2018; 37: 509-511.

[12] Marini JJ, Gattinoni L. Management of COVID-19 Respiratory Distress. Journal of the American Medical Association. 2020; 323: 2329.

[13] Mahase E. COVID-19: most patients require mechanical ventilation in first 24 hours of critical care. British Medical Journal. 2020; 368: m1201.

[14] Barr J, Fraser GL, Puntillo K, Ely EW, Gélinas C, Dasta JF, et al. Clinical practice guidelines for the management of pain, agitation, and delirium in adult patients in the intensive care unit. Critical Care Medicine. 2013; 41: 263-306.

[15] Grasselli G, Pesenti A, Cecconi M. Critical Care Utilization for the COVID-19 Outbreak in Lombardy, Italy: early experience and forecast during an emergency response. Journal of the American Medical Association. 2020; 323: 1545-1546.

[16] Sanders JM, Monogue ML, Jodlowski TZ, Cutrell JB. Pharmacologic treatments for coronavirus disease 2019 (COVID-19): A Review. Journal of the American Medical Association. 2020; 323: 1824-1836.

[17] Monedero P, Gea A, Castro P, Candela-Toha AM, Hernández-Sanz ML, Arruti E, et al. Early corticosteroids are associated with lower mortality in critically ill patients with COVID-19: a cohort study. Critical Care. 2021; 25: 2 .

[18] Lonardo NW, Mone MC, Nirula R, Kimball EJ, Ludwig K, Zhou X, et al. Propofol is associated with favorable outcomes compared with benzodiazepines in ventilated intensive care unit patients. American Journal of Respiratory and Critical Care Medicine. 2014; 189: 13831394.

[19] Skrobik Y Duprey Ms, Hill NS, Devlin G. Low-dose nocturnal dexemedetimidine prevents ICU delirium, a randomized, placebocontrolledtrial. American Journal of Respiratory and Critical Care Medicine. 2018; 197: 1147-1156.

[20] Le Guen M, Liu N, Bourgeois E, Chazot T, Sessler DI, Rouby J, et al. Automated sedation outperforms manual administration of propofol and remifentanil in critically ill patients with deep sedation: a randomized phase II trial. Intensive Care Medicine. 2013; 39: 454-462.

[21] Girard TD, Kress JP, Fuchs BD, Thomason JW, Schweickert WD, Pun BT, et al. Efficacy and safety of a paired sedation and ventilator weaning protocol for mechanically ventilated patients in intensive care (awakening 
and breathing controlled trial): a randomized controlled trial. Lancet. 2008; 371: 126-134

[22] Rubulotta F, Rubulotta G, Occhipinti G, Naimo J, Gullo A. Comment on "Effects of neuromuscular block on systemic and cerebral hemodynamics and bispectral index during moderate or deep sedation in critically ill patients" by Inoue et al. Intensive Care Medicine. 2007; 33: 388-389.

[23] Solverson K, Weatherald J, Parhar KKS. Tolerability and safety of awake prone positioning COVID-19 patients with severe hypoxemic respiratory failure. Canadian Journal of Anesthesia. 2021; 68: 64-70.

[24] Ferguson ND, Fan E, Camporota L, Antonelli M, Anzueto A, Beale R, et $a l$. The Berlin definition of ARDS: an expanded rationale, justification, and supplementary material. Intensive Care Medicine. 2012; 38: 15731582.

[25] Armstrong RA, Kane AD, Cook TM. Outcomes from intensive care in patients with COVID-19: a systematic review and meta-analysis of observational studies. Anaesthesia. 2020; 75: 1340-1349.

[26] Immovilli P, Morelli N, Antonucci E, Radaelli G, Barbera M, Guidetti D. COVID-19 mortality and ICU admission: the Italian experience. Critical Care. 2020; 24: 228.

[27] Soy M, Keser G, Atagündüz P, Tabak F, Atagündüz I, Kayhan S. Cytokine storm in COVID-19: pathogenesis and overview of anti-inflammatory agents used in treatment. Clinical Rheumatology. 2020; 39: 2085-2094.

[28] Jean S, Lee P, Hsueh P. Treatment options for COVID-19: the reality and challenges. Journal of Microbiology, Immunology and Infection. 2020; 53: 436-443

[29] McCreary EK, Angus DC. Efficacy of Remdesivir in COVID-19. Journal of the American Medical Association. 2020; 324: 1041-1042.

[30] Sinha N, Balayla G. Hydroxychloroquine and COVID-19. Postgraduate Medical Journal. 2020; 96: 550-555.

[31] Toniati P, Piva S, Cattalini M, Garrafa E, Regola F, Castelli F, et al. Tocilizumab for the treatment of severe COVID-19 pneumonia with hyperinflammatory syndrome and acute respiratory failure: A single center study of 100 patients in Brescia, Italy. Autoimmunity Reviews. 2020; 19: 102568.

[32] Connors JM, Levy JH. COVID-19 and its implications for thrombosis and anticoagulation. Blood. 2020; 135: 2033-2040.

[33] Tang N, Bai H, Chen X, Gong J, Li D, Sun Z. Anticoagulant treatment is associated with decreased mortality in severe coronavirus disease 2019 patients with coagulopathy. Journal of Thrombosis and Haemostasis. 2020; 18: 1094-1099.

[34] Saghazadeh A, Rezaei N. Towards treatment planning of COVID-19: Rationale and hypothesis for the use of multiple immunosuppressive agents: Anti-antibodies, immunoglobulins, and corticosteroids. International Immunopharmacology. 2020; 84: 106560.

[35] Hushmandi K, Bokaie S, Hashemi M, Moghadam ER, Raei M, Hashemi $\mathrm{F}$, et al. A review of medications used to control and improve the signs and symptoms of COVID-19 patients. European Journal of Pharmacology. 2020; 887: 173568 .

[36] Balas MC, Weinhouse GL, Denehy L, Chanques G, Rochwerg B, Misak $\mathrm{CJ}$, et al. Interpreting and implementing the Pain, Agitation/Sedation, Delirium, Immobility, and Sleep Disruption Clinical Practice Guideline. Critical Care Medicine. 2018; 46: 1464-1470.

[37] Navas-Blanco JR, Dudaryk R. Management of Respiratory Distress Syndrome due to COVID-19 infection. BMC Anesthesiology. 2020; 20: 177.

[38] Schwarzkopf D, Behrend S, Skupin H, Westermann I, Riedemann NC, Pfeifer R, et al. Family satisfaction in the intensive care unit: a quantitative and qualitative analysis. Intensive Care Medicine. 2013; 39 : 1071-1079.

[39] Devlin JW, Skrobik Y, Gélinas C, Needham DM, Slooter AJC,
Pandharipande PP, et al. Clinical Practice Guidelines for the Prevention and Management of Pain, Agitation/Sedation, Delirium, Immobility, and Sleep Disruption in Adult Patients in the ICU. Critical Care Medicine. 2018; 46: e825-e873.

[40] Mehta S, Spies C, Shehabi Y. Ten tips for ICU sedation. Intensive Care Medicine. 2018; 44: 1141-1143.

[41] Chanques G, Kress JP, Pohlman A, Patel S, Poston J, Jaber S, et al. Impact of Ventilator Adjustment and Sedation-Analgesia Practices on Severe Asynchrony in Patients Ventilated in Assist-Control Mode. Critical Care Medicine. 2013; 41: 2177-2187.

[42] Minhas MA, Velasquez AG, Kaul A, Salinas PD, Celi LA. Effect of Protocolized Sedation on Clinical Outcomes in Mechanically Ventilated Intensive Care Unit Patients: a Systematic Review and Meta-analysis of Randomized Controlled Trials. Mayo Clinic Proceedings. 2015; 90: 613623.

[43] Stollings J, Szumita P, Devlin J. Choice of analgesia and sedation. In Posa SP, Stollings JL (eds.) ICU liberation. Society of Critical Care Medicine, Mount Prospect. 2020.

[44] Jabaudon M, Boucher P, Imhoff E, Chabanne R, Faure JS, Roszik L, et al. Sevoflurane for sedation in acute respiratory distress syndrome: a randomized pilot study. American Journal of Respiratory and Critical Care Medicine. 2017; 195: 702-800.

[45] Alhazzani W, Belley-Cote E, Møller MH, Angus DC, Papazian L, Arabi YM, et al. Neuromuscular blockade in patients with ARDS: a rapid practice guideline. Intensive Care Medicine. 2020; 46: 1977-1986.

[46] Rose L, Fitzgerald E, Cook D, Kim S, Steinberg M, Devlin JW, et al. Clinician perspectives on protocols designed to minimize sedation. Journal of Critical Care. 2015; 30: 348-352.

[47] Aitken LM, Bucknall T, Kent B, Mitchell M, Burmeister E, Keogh S. Sedation protocols to reduce duration of mechanical ventilation in the ICU: a Cochrane Systematic Review. Journal of Advanced Nursing. 2016; 72: 261-272.

[48] Jakob SM. Dexmedetomidine vs Midazolam or Propofol for Sedation during Prolonged Mechanical Ventilation: two randomized controlled trials. Journal of the American Medical Association. 2012; 307: 11511160.

[49] Rijkenberg S, Stilma W, Endeman H, Bosman RJ, Oudemans-van Straaten HM. Pain measurement in mechanically ventilated critically ill patients: Behavioral Pain Scale versus Critical-Care Pain Observation Tool. Journal of Critical Care. 2015; 30: 167-172.

[50] Chanques G, Viel E, Constantin J, Jung B, de Lattre S, Carr J, et al. The measurement of pain in intensive care unit: comparison of 5 self-report intensity scales. Pain. 2010; 151: 711-721.

[51] Chanques G, Tarri T, Ride A, Prades A, De Jong A, Carr J, et al. Analgesia nociception index for the assessment of pain in critically ill patients: a diagnostic accuracy study. British Journal of Anaesthesia. 2017; 119: $812-820$.

[52] Pajen JF, Bosson JL, Chanques G, Mantz J, Labarere J. Pain assessment is associated with decreased duration of mechanical ventilation in the intensive care unit: a post Hoc analysis of the Dolore a study. Anesthesiology 2009; 111: 1308-1316.

How to cite this article: Caterina Aurilio, Antonio Corcione, Fiorentino Fraganza, Pasquale Sansone, Antonella Paladini, Maria Beatrice Passavanti, et al. Sedation and analgesia in critically ill patients with COVID-19: a cohort retrospective study. Signa Vitae. 2021;17(5):52-57. doi:10.22514/sv.2021.129. 